\title{
Muscle strength in overwintering bears
}

\author{
Unlike humans, bears retain their muscle tone when moribund for long periods.
}

$\mathrm{B}$ lack bears (Ursus americanus) stay inside their winter den for five to seven months of the year, during which time their body temperature drops to about $4{ }^{\circ} \mathrm{C}$ below normal ${ }^{1}$ and they do not eat, drink, urinate, defecate or show any other perceptible activity ${ }^{2}$. Although inactivity in humans, for example as a result of confined bed rest, weightlessness or limb immobilization, leads to atrophy of skeletal muscle, loss of muscle tone and impaired strength, we show here that the black bear does not suffer a similar deterioration. We find that overwintering black bears lose less than $23 \%$ of their strength over 130 days - unlike humans, who are weakened by a predicted $90 \%$ strength loss over the same period.

In rodents and humans experiencing muscle atrophy resulting from inactivity or starvation, there is a reduction in the number and size of muscle cells (fibres) ${ }^{3}$, in the ratio of slow-oxidative to fast-glycolytic fibres $^{4}$, and in the myofibrillar protein content ${ }^{5}$. We have investigated how these parameters are affected in overwintering black bears by taking muscle biopsies from denned bears during early and late winter. Remarkably, we found no loss of skeletalmuscle cell number or size. In addition, some muscles retained their protein content and oxidative capacity completely, whereas others showed a slight reduction in the ratio of the two metabolic types of fibre and in their protein content ${ }^{6}$.

In view of these findings that bear muscle shows none of the typical signs of disuse atrophy and indicating that bears are likely to retain their locomotor prowess through the winter, we tested whether their muscle strength was affected by prolonged starvation and immobility. We monitored hindleg flexion of bears in the field during late autumn, just after denning, and again in early spring before they emerged from their dens (Fig. 1). Muscle strength was measured using a modification of a noninvasive force-assessment technique that had been previously validated in a hospital setting on humans for diagnosing neuromuscular disorders, for assessing weakness due to disease progression, and for evaluating therapy ${ }^{7}$. We were able to quantify the strength of the dorsiflexor muscle (tibialis anterior) of wild bears in their natural setting by measuring muscle contraction in response to supramaximal stimulation of the common perineal nerve ${ }^{8}$.

Human strength tested on a similar apparatus registers typically as around 0.54 newton metres $(\mathrm{Nm})$, which is comparable to the strength predicted for bears by

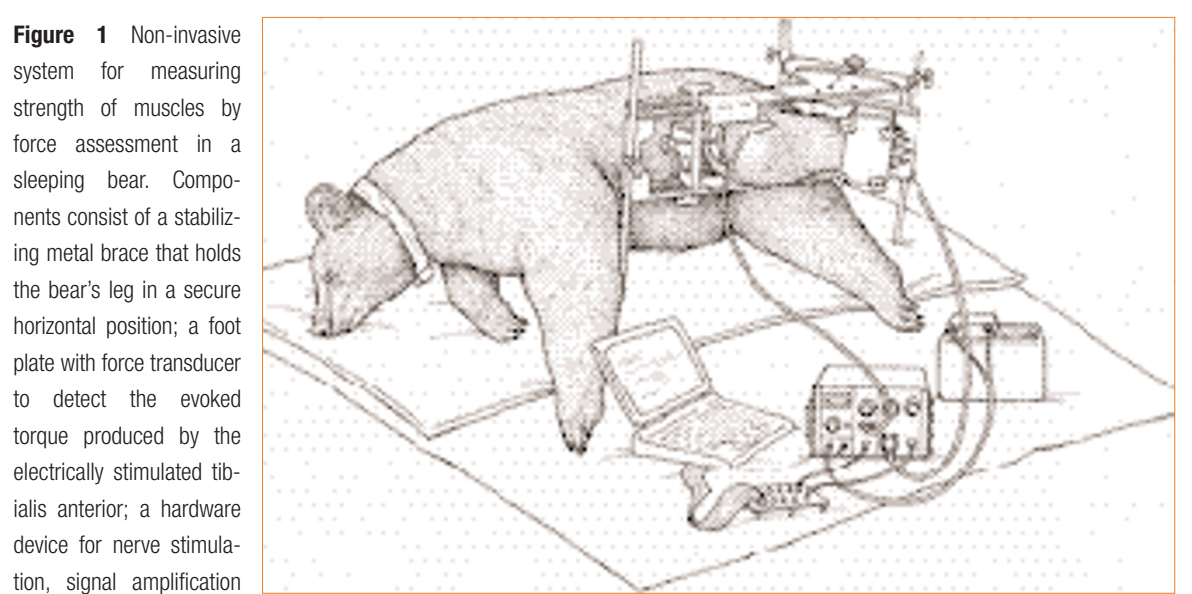
tion, signal amplification and signal conditioning, plus a computer for stimulus delivery, equipped with data-acquisition software for recording, analysing and displaying all signals simultaneously, together with a battery power-source.

extrapolation to their hibernation muscle temperature? ${ }^{9}$. Muscle force measurements in lower-limb extensor and flexor muscles of humans under conditions of confined hospital-bed rest or simulated space flight show that there is a daily loss of strength of about $0.7 \%$ as a result of limb unloading ${ }^{10}$. If strength loss as a result of atrophy were to occur at this rate in bears, a decrease in peak torque force to about $0.0675 \mathrm{Nm}$ (90\% loss of strength) would be expected after 130 days of inactivity (Fig. 2). But surprisingly, even though we found a significant decrease in torque force, bear strength remained at around $0.35 \mathrm{Nm}$ under these conditions, corresponding to a loss in strength of only $23 \%$ (Fig. 2).

Skeletal muscle protein and strength can be conserved by using alternative sources of

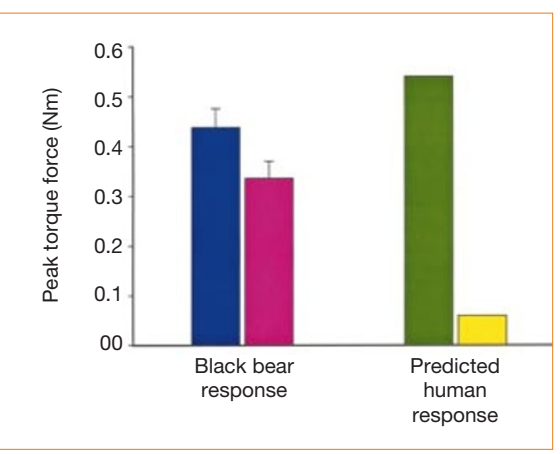

Figure 2 Muscle strength in hibernating black bears. Left, peak torque force (in newton metres, Nm) of the tibialis anterior in six bears tested in response to a multiple-pulse stimulus to the perineal nerve. Bears were tested at their den site in the autumn (blue bar) and again in the spring (pink bar); vertical lines are 1 s.e.m. Right, peak torque force $(\mathrm{Nm})$ of ankle dorsiflexion in healthy humans determined using a similar apparatus and protocol. Green bar, predicted strength at a leg temperature similar to that of the bears; yellow bar, predicted strength after 130 days of atrophy at $0.7 \%$ strength loss per day. protein, by recycling urea nitrogen back into protein synthesis, or by rhythmically stimulating the muscles. Hibernating bears may therefore be able to retain their strength by synthesizing new amino acids and protein from urea nitrogen ${ }^{11}$, or by shivering and undergoing isometric muscle contraction through the winter, or by drawing on labile protein reserves such as visceral smooth muscle and extracellular matrix. Understanding these processes in hibernating bears may provide new insight into treating muscle disorders and into the effects of prolonged hospital-bed confinement, antigravity and long-distance space travel on humans.

Henry J. Harlow ${ }^{\star}$, Tom Lohuis*, Thomas D. I. Beck $\dagger$, Paul A. Iaizzo $\ddagger$ ${ }^{*}$ Department of Zoology and Physiology, University of Wyoming, Laramie, Wyoming 82071, USA e-mail: hharlow@uwyo.edu †Colorado Division of Wildlife, 23929 Colorado Road U, Dolores, Colorado 81323, USA $\ddagger$ Departments of Anesthesiology and Physiology, University of Minnesota, Minneapolis, Minnesota 55455, USA

1. Nelson, R. A. et al. Int. Conf. Bear Res. Manag. 5, 284-290 (1983).

2. Nelson, R. A. et al. Am. J. Physiol. 224, 491-496 (1973).

3. Nicks, D. K., Beneke, W. M., Key, R. M. \& Timson, B. F. J. Anat. 163, 1-5 (1989).

4. Musacchia, X. J., Steffen, J. M. \& Deavers, D. R. Aviat. Space Environ. Med. 54, 1015-1020 (1983).

5. Booth, F. W. \& Kelso, J. R. Pflugers Arch. 342, 231-238 (1973). 6. Tinker, D. B., Harlow, H. J. \& Beck, T. D. I. Physiol. Zool. 71, 414-424 (1998).

7. Sigg, M. D., Hasinoff, I. K. \& Iaizzo, P. A. Progr. Anesthesiol. XII, 127-152 (1998).

8. Brass, T. J., Loushin, M. K. H., Day, J. W. \& Iaizzo, P. A. J. Med. Eng. Tech. 20, 67-74 (1996).

9. Quinlan, J. G., Iaizzo, P. A., Lambert, E. H. \& Gronert, G. A. Musc. Nerve 12, 119-125 (1989).

10. Berg, H. E., Larsson, L. \& Tesch, P. A. J. Appl. Physiol. 82, 182-188 (1997).

11. Nelson, R. A., Jones, J. D., Wahner, H. W., McGill, D. B. \& Code, C. F. Mayo Clin. Proc. 50, 141-146 (1975). 